\title{
Macular hole surgery using silicone oil tamponade
}

\author{
N Karia, A Laidlaw, J West, E Ezra, M Z Gregor
}

\begin{abstract}
Backgroundlaims-Most surgeons performing macular hole surgery using long acting gas recommend strict postoperative face down posturing for 10-15 days. Patients with chronic systemic illness such as arthritis may be unable to carry out this postoperative regime. Thus there is a need for alternative techniques that would eliminate such a regime. The authors review a series of patients who underwent macular hole surgery using silicone oil without any postoperative posturing.

Methods-A retrospective case note review was performed of patients who had undergone macular hole surgery with silicone oil tamponade. The patients were unable to posture due to chronic illness and had stage 2,3 , or 4 full thickness macular holes. Removal of silicone oil performed with or without cataract surgery was arranged 3 months or more after surgery.

Results-10 eyes of 10 patients underwent surgery. Duration of oil tamponade ranged from 3-9 months. Following oil removal the hole was closed in eight eyes $(80 \%)$, of which only three showed any improvement in visual acuity $(38 \%)$ even after cataract extraction. All eyes developed cataract to varying degrees and one eye developed raised intraocular pressure which settled after oil removal. A serious complication, endophthalmitis, occurred in one eye following removal of sutures after cataract extraction.

Conclusion-The anatomical results $(80 \%)$ in this series are in keeping with those reported in other studies using gas tamponade. The visual results are disappointing and less rewarding than those obtained after successful surgery using gas tamponade.
\end{abstract}

(Br f Ophthalmol 2001;85:1320-1323)

The success rates of surgery for full thickness macular holes (FTMH) have been steadily improving. The procedure as originally described $^{1}$ consists of pars plana vitrectomy, the separation of the posterior hyaloid cortex from the retinal surface, and the filling of the vitreous cavity with a long acting gas. Postoperatively, strict face down posturing for 10-15 days is recommended to ensure that the gradually shrinking gas bubble remains in contact with the FTMH.

Although most patients manage to adhere to the prolonged face down posturing regime, some are unable to do so because of systemic illness such as arthritis. Therefore, there is a need for alternative techniques which would minimise or even eliminate the need for this gruelling postoperative regime.

Silicone oil has been widely used as a surgical tool in retinal surgery for many years. ${ }^{2}$ Its advantages are that it maintains a constant volume within the vitreous cavity and the need for postoperative posturing is reduced. The few reported results of macular hole surgery using silicone oil have been contradictory. ${ }^{3}$ We therefore reviewed a series of patients who underwent macular hole surgery using silicone oil without any postoperative posturing.

\section{Patients and methods}

The patients were those who required surgery for FTMH but who were unable to posture due to spinal deformity, hiatus hernia, arthritis, or angina. Others included in the study had had previous failed surgery with gas tamponade and could not or had not complied with posturing because they found it too demanding. None of the patients had any other coexistent ocular disease and all were phakic at the time of silicone oil surgery.

All patients underwent full ophthalmic examination including slit lamp biomicroscopy and indirect funduscopy, and fluorescein angiography where warranted. The best corrected Snellen acuities were noted before and after operation. Patients with stage 2,3 , or 4 idiopathic FTMH underwent surgery. ${ }^{5}$ All eyes had a cuff of subretinal fluid around the hole.

The surgery, carried out by three surgeons, involved a three port pars plana vitrectomy and the aspiration and removal of the posterior hyaloid face from the posterior pole. Any obvious epiretinal membranes causing distortion of the macula were removed but no systematic attempt was made at the removal of the internal limiting membrane. A fluid-air exchange was performed and this was followed by air-silicone oil (1000 centistokes) exchange. The oil was injected by an automated pump activated by a foot pedal to ensure a near complete filling of the vitreous cavity. Adjunctive agents such as autologous serum or growth factors were not used. Following surgery the patients were asked to avoid the face up position but otherwise no specific posturing was required.

The eyes were examined at day 1 , week 1 , and week 6 postoperatively. The best corrected Snellen visual acuity was obtained and a $\log$ MAR transformation of the Snellen data was also made. Anatomically the results were classified as to whether the FTMH was closed with the edges invisible ("closed"), flat with no fluid cuff but with the edges visible ("flat open"), or whether it remained open with the edges elevated by a fluid cuff ("open”). 

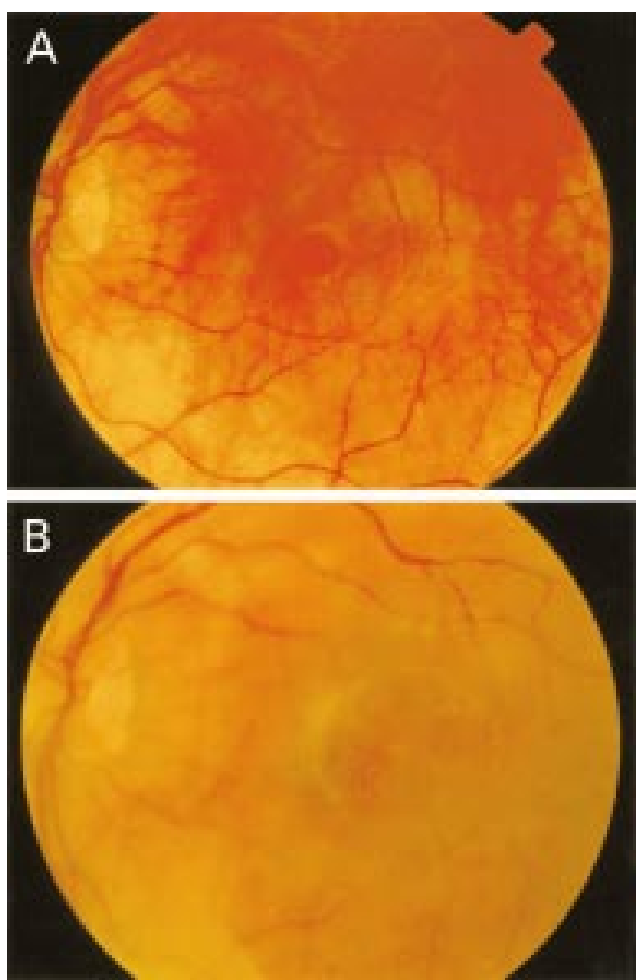

Figure 1 (A) Open macular hole before surgery. (B) Macular hole closed day 1 after operation.

Removal of silicone oil was arranged at a convenient time 3 months or more after operation. It was performed by passive efflux via a pars plana sclerostomy with the Hartmann's infusion running in simultaneously. ${ }^{6}$ When performed with cataract extraction and intraocular lens implantation the silicone oil was removed through the corneal incision. Best corrected Snellen visual acuity and the appearance of the macular hole were noted at 1 day and 6 weeks after removal of the silicone oil.

\section{Results}

There were 10 eyes of 10 patients whose age ranged from 66 years to 77 years, averaging 73 years. Three patients were male and seven were female. Duration of symptoms before surgery ranged from 7 to 22 months (average 12 months). Two eyes had stage II and six eyes

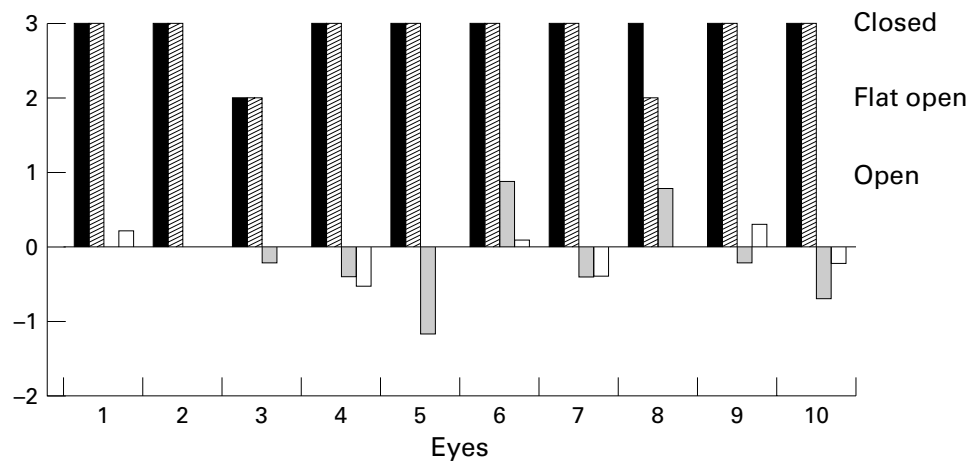

$\begin{array}{ll}\text { Hole at } 6 \text { weeks } & \text { Hole at end } \\ \text { logMAR gain at 6/52 } & \square \text { Final logMAR gain }\end{array}$

Figure 2 Anatomical and visual results at 6 weeks following macular hole surgery and finally after oil removal.
Table 1 Snellen transformation to $\log M A R$ acuity

\begin{tabular}{ll}
\hline Snellen acuity & $\log M A R$ acuity \\
\hline $1 / 60$ & 1.78 \\
$6 / 60$ & 1 \\
$6 / 36$ & 0.78 \\
$6 / 24$ & 0.6 \\
$6 / 18$ & 0.48 \\
\hline
\end{tabular}

had stage III FTMH. Two eyes with stage IV FTMH had undergone previous macular hole surgery with gas tamponade.

The silicone oil surgery proceeded without complication in all patients. Seven of the 10 eyes also had removal of epiretinal membrane. On day 1 following surgery the holes were noted to be closed in four eyes (see Fig 1) and open in five. No comment was made to the appearance of the hole in one eye. All eyes were commented on to have a good oil fill.

The duration of oil tamponade ranged from 3-9 months (mean 5.7 months). In eight eyes cataract extraction and intraocular lens implantation was carried out concurrently with removal of oil. In one eye this was done 6 months following oil removal. One eye (number 10, Fig 2) is still phakic. Follow up after oil removal ranged from 1 to 20 months (average 9 months).

\section{ANATOMICAL RESULTS}

At 6 weeks following silicone oil injection the FTMH was closed in nine eyes and flat open in one eye (Fig 2). Following oil removal the hole remained closed in eight eyes $(80 \%$ final closure rate). It became flat open after having been closed in one patient (number 8, Fig 2). An epiretinal membrane had not been removed from this eye but had been removed from seven of the eight eyes in which the hole remained closed. Eyes number 5 and 9 (Fig 2) were stage 4 holes both of which remained closed. No cystoid macular oedema was observed in any of the eyes studied.

VISUAL RESULTS

Figure 2 shows the change in the Snellen transformed $\log$ MAR visual acuity of the patients at 6 weeks following the silicone oil injection and at 6 weeks following the removal of silicone oil compared with preoperative measurements. A 0.3 gain in $\log M A R$ acuity represents a doubling of the minimum visual angle of resolution (and hence a worsening in visual acuity, see Table 1). At 6 weeks six of the nine eyes in which the hole was closed $(67 \%)$ showed an improvement in Snellen visual acuity by at least one line and four eyes (45\%) improved by two lines or more. Following oil removal only three out of the eight eyes $(38 \%)$ in which the hole remained closed maintained an improved visual acuity. The remainder showed no change or a slight gain in the $\log$ MAR acuity compared with preoperative measurements.

\section{COMPLICATIONS}

All phakic eyes developed varying degrees of lens opacity following oil tamponade and required cataract surgery. Eye number 3 (Fig 
2), in which the hole remained flat open, developed raised intraocular pressure which settled following oil removal. Eye number 5 (Fig 2), developed endophthalmitis following removal of corneal sutures after cataract extraction and intraocular lens implantation. Although the hole remained sealed there was a fall in visual acuity back to preoperative levels.

\section{Discussion}

A pilot study has suggested that postoperative face down posturing may be unnecessary in pseudophakic eyes if attention is paid in obtaining a complete gas fill of non-expansile $15 \%$ perfluoropropane gas. ${ }^{7}$ However, the flotation force of the gas bubble is greatest at its apex and face down positioning with a large gas bubble results in a greater flotation force on the macular hole than upright positioning. ${ }^{8}$ This is thought to enhance the tamponading effect of the gas bubble and allow Muller cell processes and glial cells to form a stable plug within the hole $^{910}$ which leads to reapproximation of the edges of the hole and its closure. It also facilitates an adhesion of the edges of the hole to the underlying retinal pigment epithelium. The presence of fluid in the early postoperative period can interfere with these processes and a longer duration of intraocular gas tamponade has been found to correlate with a higher rate of macular hole closure. ${ }^{8}$

In the upright position a long acting gas bubble (for example, $16 \% \mathrm{C}_{3} \mathrm{~F}_{8}$ ) will maintain a tamponade of the macular hole for approximately 2 weeks. ${ }^{8}$ After this the bubble meniscus fails to cover the macular hole. The flotation forces of the smaller intraocular bubble will also be less. Hence most surgeons feel that, in addition to an adequate gas fill, face down posturing in the early postoperative period is essential for a successful anatomical outcome to macular hole surgery. The sustained face down posturing is demanding for the patients and some may fail to comply with it. Others are unable to posture because of preexisting medical conditions. In such a group of patients macular hole surgery using gas tamponade is less likely to be successful.

The density of silicone oil is 3\% less than that of water. Thus the intraocular oil bubble will float to the top but the flotation force is only $1 / 30$ of the upward force caused by a gas bubble. ${ }^{11}$ Volume displacement is much more essential to the effects of the silicone oil bubble inside the eye. Hence the success of oil as a tamponading agent is probably due to the prevention of any fluid reaching the hole rather than the force with which it holds the retina against the pigment epithelium. The completeness of oil fill of the vitreous cavity has certainly been found to affect the seal rate of the macular holes. ${ }^{3}$ However, for the same percentage of fill the arc of contact of a bubble of oil with the retina has been found to be about half that of gas with retina. ${ }^{12}$ Hence silicone oil may have inherent "anti-wetting" properties which may also help explain its good tamponading properties. This may reflect in our observation of some of the holes being closed invisible day 1 postoperatively on slit lamp biomicroscopy.
However, comparisons with gas tamponade cannot be made owing to the poor fundal view afforded by an intraocular gas bubble.

Although the number of patients in this series is relatively small the seal rate of macular holes in this study $(80 \%)$ compares well with those reported in other studies using gas tamponade. ${ }^{813-15}$ Our rate is also comparable with the $80 \%$ success rate reported by Goldbaum et al. ${ }^{3}$ Bopp $^{4}$ achieved a closure rate of $91 \%$ using silicone oil tamponade.

However, although the anatomical results of surgery are good the final visual results in our series seem less rewarding than those obtained after successful FTMH surgery using gas tamponade even following cataract extraction. This is in keeping with Bopp's series ${ }^{4}$ where only $25 \%$ of 35 eyes achieved an improvement in vision of at least two lines. On the other hand Goldbaum et $a l^{3}$ with an average follow up of 10.3 months following oil removal, report better results with visual acuity increasing by more than 1 line on the ETDRS chart in $72 \%$ of eyes with sealed holes. However, only $56 \%$ attained $20 / 50$ or better visual acuity. In comparison Pearce $e t a l,{ }^{15}$ in their series with gas tamponade, report an improvement of at least two Snellen lines in $60 \%$ of eyes with closed holes at 3 months. Using autologous serum as adjunctive therapy and gas tamponade in 43 eyes Wells and Gregor $^{16}$ report an improvement in vision in about one half of the eyes. Twenty eyes achieved a visual acuity of $6 / 12(20 / 40)$ or better. In the study by Freeman et $a l^{13}$ using gas tamponade $48 \%$ of eyes in which the hole closed showed improvement in vision by at least one line on the ETDRS test at 6 months.

There are several possible reasons for the seemingly worse visual outcome than those obtained with gas tamponade. Furthermore, the duration of the macular holes (or the symptoms) before surgery may also influence the visual outcome after surgery. In our series this ranged from 7 to 22 months with an average of 12 months whereas in Goldbaum's series $^{3}$ where better visual results were reported the average was 5.6 months (range 0.7 to 22 months). Using gas tamponade Tornambe et $a l^{7}$ and Leonard et $a l^{17}$ report good visual results. However, whereas hole duration in Leonard's series ${ }^{17}$ ranged from 1 week to 13 months (average 5.6 months), that in Tornambe's series ${ }^{7}$ ranged from 1 month to 10 years with over $35 \%$ of holes being present for more than 1 year. It may be that silicone oil is toxic and retards photoreceptor recovery. This may help explain why our visual outcomes were better at 6 weeks and deteriorated following oil removal after a longer period of oil tamponade. Bopp et $a l^{4}$ found that droplets of silicone oil became permanently incorporated into the superficial layers of the retina. They believed that these droplets were responsible for the poor postoperative visual results. If silicone oil is used for macular hole surgery it may warrant very early removal.

Cataract formation is almost universal with macular hole surgery and this will impede visual acuity. Leonard et $a l^{17}$ report an immediate improvement in visual acuity after cataract 
surgery in patients with successful macular hole surgery having used gas tamponade. However, nine of the 10 eyes in our study were pseudophakic at follow up; this figure is unclear in Goldbaum's study. ${ }^{3}$ With long term follow up of a large percentage of their patients Leonard $e t a l^{17}$ also found that the visual acuity continued to improve after as long as $2-3$ years after macular hole surgery. They suggest that centripetal relocation of photoreceptors and differences in their recovery rates may help account for this progressive visual improvement. Whether this continued visual improvement also occurs after the use of oil tamponade remains to be seen.

The work was presented as a poster at the British Ophthalmology Congress in Cardiff, 1999.

1 Kelly NE, Wendel RT. Vitreous surgery for idiopathic macular holes. Results of a pilot study. Arch Ophthalmo 1991;109:654-9.

2 Capone A Jr, Aaberg TM. Silicone oil in vitreoretinal surgery. Curr Opin Ophthalmol 1995;6:33-7.

3 Goldbaum MH, McCuen BW, Hanneken AM, et al. Silicone oil tamponade to seal macular holes without position restrictions. Ophthalmology 1998;105:2140-7.

4 Bopp S, personal communication. Comment: DuBosar R. Physician encourages gas tamponade. Ocular Surgery News 1999; May:35.

5 Johnson RN, Gass JDM. Idiopathic macular holes. Observations, stages of formation, and implications for surgical tions, stages of formation, and implications
intervention. Ophthalmology 1988;95:917-24. 6 Leaver PK, Lean JS. Management of giant retinal tears using vitrectomy and silicone oil/fluid exchange. A prelimi-

7 Tornambe PE, Poliner LS, Grote K. Macular hole surgery without face down positioning. A pilot study. Retina 1997; 17:179-85.

8 Thompson JT, Smiddy WE, Glaser BM, et al. Intraocular tamponade duration and success of macular hole surgery. Retina 1996;16:373-82.

9 Madreperla SA, Geiger GL, Funata M, et al. Clinicopathologic correlation of a macular hole treated by cortical
vitreous peeling and tamponade. Ophthalmology 1994;101: vitreous

10 Funata M, Wendel RT, de la Cruz Z, et al. Clinicopathologic study of bilateral macular holes treated with pars plana vitrectomy and gas tamponade. Retina 1992;12:289-98.

11 Peterson J. The physical and surgical aspects of silicone oil in the vitreous cavity. Graefes Arch Clin Exp Ophthalmol 1987;225:452-6.

12 Fawcett IM, Williams RL, Wong D. Contact angles of substances used for internal tamponade in retinal detachment surgery. Graefes Arch Clin Exp Ophthalmol 1994;232: 438-44

13 Freeman WR, Azen SP, Kim JW, et al. Vitrectomy for the treatment of full thickness stage 3 or 4 macular holes. Results of a multicentered randomised clinical trial. The vitrectomy for treatment of macular hole study group. Arch Ophthalmol 1997;115:11-21.

14 Ruby AJ, Williams DF, Grand MG, et al. Pars plana vitrectomy for treatment of stage 2 macular holes. Arch Ophthalmol 1994;112:359-64.

15 Pearce IA, Branley M, Groenewald C, et al. Visual function and patient satisfaction after macular hole surgery. Eye 1998;12(pt 4):651-8.

16 Wells JA, Gregor ZJ. Surgical treatment of full thickness macular holes using autologous serum. Eye 1996;10(pt 5):593-9.

17 Leonard RE, Smiddy WE, Flynn HW, et al. Long term visual outcomes in patients with successful macular hole surgery. Ophthalmology 1997;104:1648-52. 